\title{
Impressive Long-term Response with Pertuzumab and Trastuzumab in HER2-positive Breast Cancer with Brain Metastasis
}

\author{
MICHELANGELO RUSSILLO ${ }^{1}$, GIANLUIGI FERRETTI ${ }^{1}$, ANTONELLO VIDIRI ${ }^{2}$, SIMONA GASPARRO ${ }^{1}$, \\ FRANCESCO COGNETTI ${ }^{1}$, DOMENICA PELLEGRINI ${ }^{1}$ and ALESSANDRA FABI ${ }^{1}$ \\ ${ }^{1}$ Division of Medical Oncology 1, Regina Elena National Cancer Institute, Rome, Italy; \\ ${ }^{2}$ Department of Neuroscience, Imaging Diagnostic, Regina Elena National Cancer Institute, Rome, Italy
}

\begin{abstract}
This is a case report of a 40-year-old woman who, after conservative breast cancer treatment, developed a HER2 positive solitary brain metastasis in the left temporal lobe, without extracranial disease. She underwent surgery resection followed by stereotactic radiotherapy and, because of early brain progression, she was submitted to the first line therapy with pertuzumab, trastuzumab and weekly paclitaxel. After six months of treatment, a brain magnetic resonance imaging revealed a complete disappearance of brain recurrence, which persisted for more than 24 months.
\end{abstract}

In human epidermal growth factor receptor 2 (HER2)expressing breast cancer (BC) subtype the incidence of brain metastases (BM) after the diagnosis of the primary tumor is between $3.2 \%$ and $3.7 \%$ and significantly raises $(30-50 \%)$ in patients who do not receive adjuvant anti-HER2 monoclonal antibody trastuzumab $(\mathrm{T})(1,2)$.

It has been reported that, although $\mathrm{T}$ significantly improves the prognosis of patients with HER2-positive advanced BC (3), the overall survival from the time of diagnosis in women with $\mathrm{BC}$ and $\mathrm{BM}(\mathrm{BCBM})$ is less than 1.2 years $(4,5)$.

Recently, according to the Cleopatra trial, the combination of pertuzumab (P) and $\mathrm{T}$ plus docetaxel showed a significant impact on progression-free survival and overall survival in HER2-positive metastatic BC patients (6). Although this trial did not enroll BCBM patients, an exploratory analysis showed that time to BM development as the first site of

This article is freely accessible online.

Correspondence to: Alessandra Fabi, MD, Division of Medical Oncology, Regina Elena National Cancer Institute, Via Elio Chianesi 53, 00126 Rome, Italy. Tel: +39 0652665144, Fax: +39 52665637, e-mail: alessandra.fabi@virgilio.it

Key Words: Pertuzumab, metastatic breast cancer, brain metastasis. disease progression and overall survival were significantly prolonged in patients receiving $\mathrm{P}$ plus $\mathrm{T} v s . \mathrm{T}$ alone (respectively, $15 v s .11 .9$ months $p=0.0049$, and $34.4 v s .26 .3$ months $p=0.11$ ) (7). These findings are one of the main reasons to treat $\mathrm{BCBM}$ patients with the dual blockade plus taxanes as a first-line option.

This is a case report of a HER2-positive BC woman with $\mathrm{BM}$ as a unique site of disease progression. This patient experienced an impressive clinical benefit by anti-HER2 dual blockade.

\section{Case Report}

On November 2009, a 40-year old woman presented with a $20 \times 20 \mathrm{~mm}$ mass in her left breast, with palpable fixed axillary nodes. A core needle biopsy of the breast tumor revealed an invasive ductal carcinoma and the fine needle aspiration of an axillary node documented metastasis from BC. Total body Computed Tomography (CT scan) showed absence of distant metastases. The clinical stage at diagnosis was cT2N2M0. The immunohistochemical analysis revealed that the tumor expressed estrogen receptor (ER, 50\%), but not progesterone receptor (PGR, 0\%) and HER2 (0\%).

She received neo-adjuvant chemotherapy including 4 courses of 3-weekly doxorubicin $60 \mathrm{mg} / \mathrm{m}^{2}$ plus cyclophosphamide $600 \mathrm{mg} / \mathrm{m}^{2}$ followed by 4 courses of 3 weekly docetaxel $100 \mathrm{mg} / \mathrm{m}^{2}$. A radical mastectomy with axillary node dissection was subsequently performed. The histological examination revealed a pathological partial response with residual ductal infiltrating carcinoma in the left breast and absence of metastases in the axillary nodes (pT1bN0). The biological profile was: ER 55\%, PgR 25\%, HER2-negative and Ki-67 15\%. The patient received antiestrogen treatment with tamoxifen plus triptorelin until November 2014.

On October 2015, after persistent headache, she underwent total body $\mathrm{CT}$ scan and brain magnetic resonance 
A

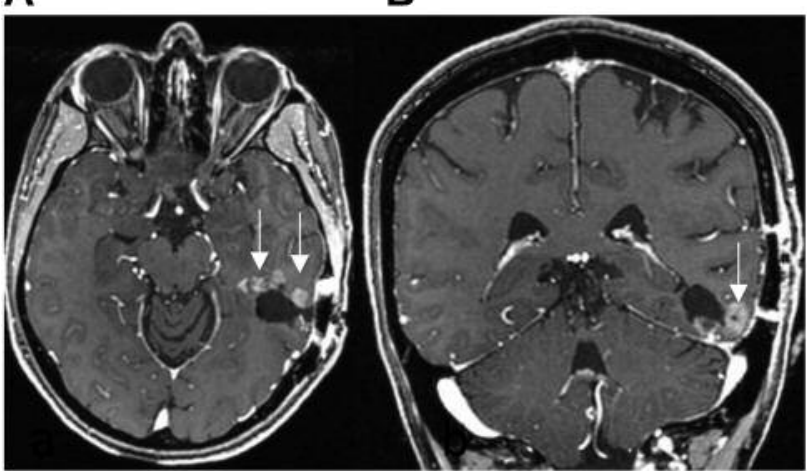

Figure 1. MRI images with SE T1 after gadolinium in axial (A) and coronal $(B)$ planes show peripherally at surgical cavity the presence of small nodular lesions (arrows) in relation to recurrence.

imaging (MRI): a single BM in the left temporal lobe was discovered, without extracranial diffusion of the disease. A surgical resection of the right temporal metastasis was performed and the histological examination confirmed the origin from breast carcinoma, with the following biological features: ER 55\%, PGR 20\%, HER2 2+, SISH amplified. On November 2015, the woman received 4 courses of stereotactic brain radiotherapy in the site of surgery. After 40 days from surgery, the brain MRI revealed the presence of a small nodular recurrence close to the surgical cavity (Figure $1 \mathrm{~A}$ and $\mathrm{B})$. The patient did not complain of any neurological symptoms. Again, the total body CT scan showed a absence of extra-cranial diffusion of the disease.

Between December 2015 and May 2016, the patient received 8 cycles of $\mathrm{T}$ ( $8 \mathrm{mg} / \mathrm{kg}$ iv loading dose followed by 6 $\mathrm{mg} / \mathrm{kg}$ every three weeks), $\mathrm{P}$ (840 $\mathrm{mg}$ iv loading dose followed by $420 \mathrm{mg}$ every three weeks) and weekly paclitaxel (80 $\mathrm{mg} / \mathrm{m}^{2}$ ) over a period of six months for a total of 24 weekly administrations. The therapy was well tolerated without serious adverse events. During the course of combined treatment, the patient had brain MRI every 3 months which showed a progressive shrinkage of BM (Figure $2 \mathrm{~A}$ and B), while the CT scan did not document extracranial metastases. From June 2016 the patient has been receiving maintenance treatment with the dual monoclonal blockade. She has an optimal performance status, without any neurological symptom. The last brain MRI has confirmed the persistence of a complete response obtained from May 2016 (Figure 2C and D).

\section{Discussion}

The management of patients with BCBM include both surgery and radiotherapy (RT), which are the standard firstline modalities for treatment of BM.
A

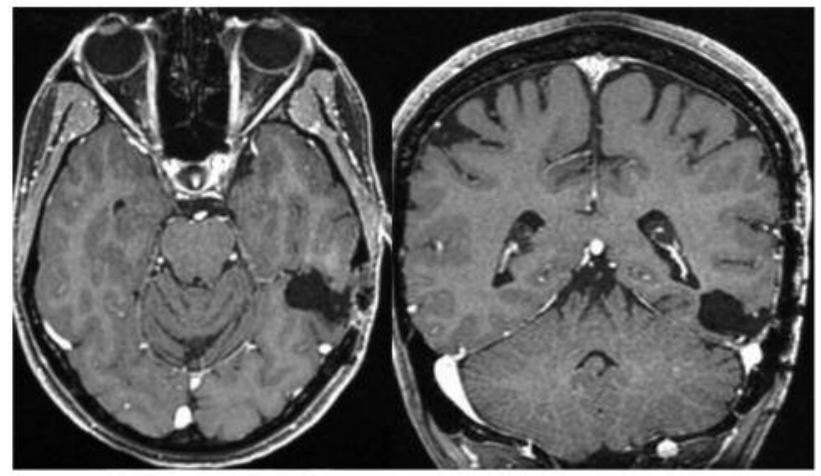

C

D

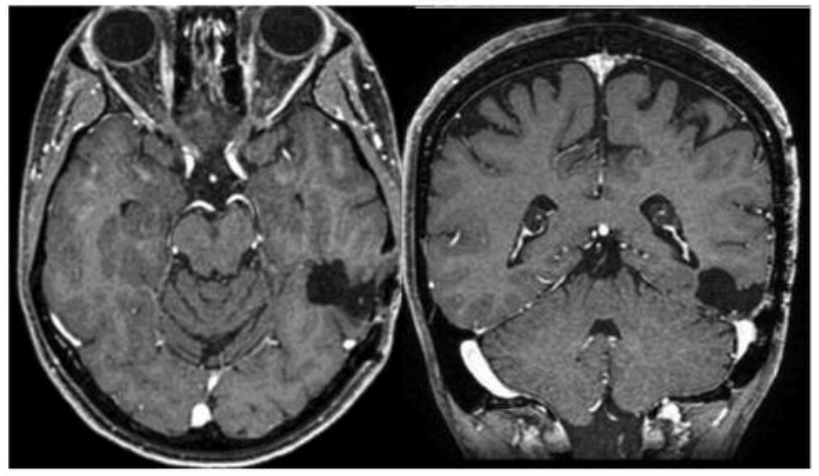

Figure 2. MRI images with SE T1 after gadolinium in axial (A) and coronal (B) planes after 12 months and in axial $(C)$ and coronal (D) planes after 24 months, show disappearance of the nodular lesions, in relation to complete response to treatment.

Whole brain radiotherapy (WBRT) is recommended for the treatment of multiple BM while stereotactic radiosurgery (SRS) is an option in patients with oligometastatic disease.

Systemic therapy plus $\mathrm{T}$, administered after WBRT, appears to have a positive impact on survival among HER2 positive $\mathrm{BC}$ patients with $\mathrm{BM}$, probably due to the control of extracranial disease (8).

Pertuzumab is a humanized monoclonal antibody which binds to the subdomain II dimerization arm of HER2 receptor and inhibits the interaction of HER2 with its main co-receptor HER3 (9). As well as T, P stimulates antibodydependent and cell-mediated cytotoxicity. In HER2-positive preclinical tumor models the combination of $\mathrm{T}$ plus $\mathrm{P}$ resulted in greater antitumor inhibition than either agent alone (10). On this basis the efficacy and safety of P plus T were evaluated in phase II and III clinical trials carried out in patients with HER2-positive metastatic BC (11-13). The impressive findings of the registrative CLEOPATRA study with the significantly prolonged progression free survival and overall survival in favour of dual blockade with $\mathrm{P}+\mathrm{T}$ and 
docetaxel doubtless defined the standard of care in first-line metastatic BC (12).

Recently, Dang et al. demonstrated that paclitaxel given once per week with $\mathrm{T}$ and $\mathrm{P}$ is highly effective and welltolerated and could be an alternative to docetaxel-based combination therapy in first and second line setting (14).

No data are available on the ability of $\mathrm{P}$ to cross the blood brain barrier (BBB), but the delay in the involvement of the central nervous system, as shown by Swain et al., leads to the hypothesis of a possible synergistic activity of $\mathrm{P}$ plus $\mathrm{T}$ on central nervous system (CNS) metastases (7).

In the second setting, recent trials evaluated BMBC patient's outcome with HER2 directed agents after T failure. Trastuzumab emtansine (T-DM1) is an antibody-drug conjugate of the anti-microtubule emtansine and $\mathrm{T}$.

In the EMILIA study, in which T-DM1 was compared with lapatinib plus capecitabine in advanced HER2 positive metastatic BC pre-treated with Trastuzumab, TDM-1 conferred a prolonged overall survival also in patients with pre-existing BCBM (26.8 vs. 12.9 months, $p=0.008$ ) (15).

The activity of other HER2-directed drugs (lapatinib and neratinib) have been also investigated in BMBC patients.

Lapatinib, a reversible dual inhibitor of the intracellular tyrosine kinase domain of HER 1 and HER2, is expected to cross the $\mathrm{BBB}$ due to its very low molecular weight. Its efficacy, has been investigated in combination with capecitabine in a small cohort of 44 HER2-positive untreated metastatic BMBC patients. In this single arm phase II trial (Landscape study), objective CNS response rate was of $65.9 \%$ $(95 \% \mathrm{CI}=50.1-79.5)$ and all were partial responses (16).

In the CEREBEL phase III trial, 540 metastatic BC patients were randomly assigned $(1: 1)$ to receive lapatinibcapecitabine or trastuzumab-capecitabine; the trial was not conclusive in terms of the incidence of CNS metastases as first site of relapse which was the primary end point of the study $(3 \%$ in the patients in the lapatinib-capecitabine arm and $5 \%$ in the T-capecitabine arm, 21.6\%; 95\% CI=22-25\%; $p=5.360)$ (17).

Neratinib, an irreversible pan-ERBB tyrosine kinase inhibitor, in combination with paclitaxel was recently compared with $\mathrm{T}$ plus paclitaxel, as first-line treatment of metastatic HER2-positive BC. In this randomized, controlled, open-label trial (NEFERT-T), neratinib plus paclitaxel was not superior to T-paclitaxel in terms of PFS [12.9 months (95\% CI=11.1-14.9) and 12.9 months $(95 \% \mathrm{CI}=11.1-14.8)$, respectively $(p=0.89)]$. However, the incidence of BM was lower (relative risk, $0.48 ; 95 \% \mathrm{CI}=0.29-0.79 ; p=0.002$ ) and the time to $\mathrm{CNS}$ metastasis was delayed $(\mathrm{HR}=0.45$; $95 \% \mathrm{CI}=0.26-0.78 ; p=0.004)$ in the experimental arm (18).

Despite the paucity of data available on the activity of the new anti-HER2 compounds in the subgroup of HER2positive $\mathrm{BC}$ women who developed $\mathrm{BM}$ as a first and single site of disease progression, our patient was treated with $\mathrm{P}$ plus $\mathrm{T}$ and paclitaxel for an early brain progression after loco-regional therapy (surgery and stereotactic radiation). A long duration of complete response in the BM (20+ months) was achieved. In this case, both the capacity of metastases itself to break the BBB facilitating the entrance of therapeutic agents and the previous surgery, which may have induced an alteration of BBB increasing the penetration of the two monoclonal antibodies, could explain the success in controlling the growth of BMs (19). In support of our findings, Mounsey et al., recently reported a longer overall survival from BCBM diagnosis among patients who received HER2 targeted therapy compared to those who did not $(2.1$ years $[95 \% \mathrm{CI}=1.6-2.6]$ vs. 0.65 years [95\%CI=0.4-1.3; $p=0.001)$ (20). The same positive impact on survival was also observed among HER2-positive BC patients who received targeted therapies after local treatment for solitary breast cancer BM (21). Finally, our findings could encourage clinicians to use the anti-HER2 double blockade for BMs even before loco-regional approaches (surgery, stereotactic RT, surgery plus stereotactic RT), especially in cases of single or oligo asymptomatic BMs, without extracranial disease. This therapeutic approach could help clinicians to eventually delay toxicities deriving from loco-regional therapies, such as (neurocognitive impairment, radionecrosis).

\section{References}

1 Pestalozzi BC, Holmes E, de Azambuja E, Metzger-Filho O, Hogge L, Scullion M, Láng I, Wardley A, Lichinitser M, Sanchez RI, Müller V, Dodwell D, Gelber RD, Piccart-Gebhart MJ and Cameron D: CNS relapses in patients with HER2positive early breast cancer who have and have not received adjuvant trastuzumab: a retrospective substudy of the HERA trial (BIG 1-01). Lancet Oncol 14: 244-248, 2013.

2 Arvold ND, Oh KS, Niemierko A, Taghian AG, Lin NU, AbiRaad RF, Sreedhara M, Harris JR and Alexander BM: Brain metastases after breast-conserving therapy and sistemic therapy: incidence and characteristics by biologic subtype. Breast Cancer Res Treat 136: 153-160, 2012.

3 Willems A, Gauger K, Henrichs C and Harbeck N: Antibody therapy for breast cancer. Anticancer Res 25: 1483-1489, 2005.

4 Bendell JC1, Domchek SM, Burstein HJ, Harris L, Younger J, Kuter I, Bunnell C, Rue M, Gelman R and Winer E: Central nervous system metastases in women who receive trastuzumabbased therapy for metastatic breast carcinoma. Cancer 97: 29722977, 2003

5 Anders CK, Deal AM, Miller CR, Khorram C, Meng H, Burrows E, Livasy C, Fritchie K, Ewend MG, Perou CM and Carey LA: The prognostic contribution of clinical breast cancer subtype, age, and race among patients with breast cancer brain metastases. Cancer 117: 1602-1611, 2011.

6 Swain SM, Baselga J, Kim SB, Ro J, Semiglazov V, Campone M, Ciruelos E, Ferrero JM, Schneeweiss A, Heeson S, Clark E, Ross G, Benyunes MC and Cortés J: Pertuzumab, trastuzumab, and docetaxel in HER2-positive metastatic breast cancer. N Engl J Med 372: 724-734, 2015. 
7 Swain SM, Baselga J, Miles D, Im YH, Quah C, Lee LF and Cortés J: Incidence of central nervous system metastases in patients with HER2-positive metastatic breast cancer treated with pertuzumab, trastuzumab, and docetaxel: results from the randomized phase III study CLEOPATRA. Ann Oncol 25: 11161121,2014

8 Niwińska A, Murawska $M$ and Pogoda K: Breast cancer subtypes and response to systemic treatment after whole-brain radiotherapy in patients with brain metastases. Cancer 116: 4238-4247, 2010

9 Lee-Hoeflich ST, Crocker L, Yao E, Pham T, Munroe X, Hoeflich KP, Sliwkowski MX and Stern HM: A central role for HER3 in HER2-amplified breast cancer: implications for targeted therapy. Cancer Res 68: 5878-5887, 2008.

10 Scheuer W1, Friess T, Burtscher H, Bossenmaier B, Endl J and Hasmann M: Strongly enhanced antitumor activity of trastuzumab and pertuzumab combination treatment on HER2-positive human xenograft tumor models. Cancer Res 69: 9330-9336, 2009.

11 Baselga J, Gelmon KA, Verma S, Wardley A, Conte P, Miles D, Bianchi G, Cortes J, McNally VA, Ross GA, Fumoleau P and Gianni L: Phase II trial of pertuzumab and trastuzumab in patients with human epidermal growth factor receptor 2- positive metastatic breast cancer that progressed during prior trastuzumab therapy. J Clin Oncol 28: 1138-1144, 2010.

12 Baselga J, Cortés J, Kim SB, Im SA, Hegg R, Im YH, Roman L, Pedrini JL, Pienkowski T, Knott A, Clark E, Benyunes MC, Ross G and Swain SM: CLEOPATRA Study Group. Pertuzumab plus trastuzumab plus docetaxel for metastatic breast cancer. N Engl J Med 366: 109-119, 2012.

13 Barthélémy P, Leblanc J, Goldbarg V, Wendling F and Kurtz JE: Pertuzumab: development beyond breast cancer. Anticancer Res 34: 1483-1491, 2014.

14 Dang C, Iyengar N, Datko F, D'Andrea G, Theodoulou M, Dickler M, Goldfarb S, Lake D, Fasano J, Fornier M, Gilewski T, Modi S, Gajria D, Moynahan ME, Hamilton N, Patil S, Jochelson M, Norton L, Baselga J and Hudis C: Phase II study of paclitaxel given once per week along with trastuzumab and pertuzumab in patients with human epidermal growth factor receptor 2-positive metastatic breast cancer. J Clin Oncol 33: 442-447, 2015.

15 Krop IE, Lin NU, Blackwell K, Guardino E, Huober J, Lu M, Miles D, Samant M, Welslau M and Diéras V: Trastuzumab emtansine (T-DM1) versus lapatinib plus capecitabine in patients with HER2-positive metastatic breast cancer and central nervous system metastases: a retrospective, exploratory analysis in EMILIA. Ann Oncol 26: 113-119, 2015.
16 Bachelot T, Romieu G, Campone M, Diéras V, Cropet C, Dalenc F, Jimenez M, Le Rhun E, Pierga JY, Gonçalves A, Leheurteur M, Domont J, Gutierrez M, Curé H, Ferrero JM, Labbe and Devilliers C: Lapatinib plus capecitabine in patients with previously untreated brain metastases from HER2-positive metastatic breast cancer (LANDSCAPE): a single-group phase 2 study. Lancet Oncol 14: 64-71, 2013.

17 Pivot X, Manikhas A, Żurawski B, Chmielowska E, Karaszewska B, Allerton R, Chan S, Fabi A, Bidoli P, Gori S, Ciruelos E, Dank M, Hornyak L, Margolin S, Nusch A, Parikh R, Nagi F, DeSilvio M, Santillana S, Swaby RF and Semiglazov V: CEREBEL (EGF111438): A Phase III, randomized, openlabel study of lapatinib plus capecitabine versus trastuzumab plus capecitabine in patients with Human Epidermal Growth Factor Receptor 2-positive metastatic breast cancer. J Clin Oncol 33: 1564-1573, 2015.

18 Awada A, Colomer R, Inoue K, Bondarenko I, Badwe RA, Demetriou G, Lee SC, Mehta AO, Kim SB, Bachelot T, Goswami C, Deo S, Bose R, Wong A, Xu F, Yao B, Bryce R and Carey LA: Neratinib Plus Paclitaxel vs. Trastuzumab Plus Paclitaxel in Previously Untreated Metastatic ERBB2-Positive Breast Cancer: The NEfERT-T Randomized Clinical Trial. JAMA Oncol 2: 1557-1564, 2016.

19 Mehta AI, Brufsky AM and Sampson JH: Therapeutic approaches for HER2-positive brain metastases: circumventing the blood-brain barrier. Cancer Treat Rev 39: 261-269, 2013.

20 Mounsey LA, Deal AM, Keith KC, Benbow JM, Shachar SS, Zagar T, Dees EC, Carey LA, Ewend MG and Anders CK: Changing Natural History of HER2-Positive Breast Cancer Metastatic to the Brain in the Era of New Targeted Therapies. Clin Breast Cancer 18: 29-37, 2018.

21 Niwińska A: Brain metastases as site of first and isolated recurrence of breast cancer: the role of systemic therapy after local treatment. Clin Exp Metastasis 33: 677-685, 2016.
Received March 15, 2018

Revised April 3, 2018

Accepted April 10, 2018 